\title{
Erratum to: Integrable QFT and Longo-Witten Endomorphisms
}

\author{
Marcel Bischoff and Yoh Tanimoto
}

\section{Erratum to: Ann Henri Poincaré (2015) 16(2):569-608 DOI 10.1007/s00023-014-0337-1}

In the original publication of the article, the authors had introduced left-right S-matrices. One of their defining properties should be corrected as follows.

On page 580, Definition 3.4(4), the right mixed Yang-Baxter equation (YBE) should be

$$
R_{23}^{-} S_{12} S_{13}=S_{13} S_{12} R_{23}^{-} .
$$

Accordingly, on page 581, Proposition 3.5(3), the claim should be

$$
\begin{aligned}
\underline{R}^{-}\left(q-q^{\prime}\right)_{23} \cdot \underline{S}(q)_{12} \cdot \underline{S}\left(q^{\prime}\right)_{13} & =\underline{S}\left(q^{\prime}\right)_{13} \cdot \underline{S}(q)_{12} \cdot \underline{R}^{-}\left(q-q^{\prime}\right)_{23}, \\
\text { i.e. } \sum_{\alpha^{\prime} \beta^{\prime} \gamma^{\prime}} \underline{R}_{\beta^{\prime} \gamma^{\prime}}^{-\beta \gamma}\left(q-q^{\prime}\right) \underline{S}_{\alpha^{\prime} \beta^{\prime \prime}}^{\alpha \beta^{\prime}}(q) \underline{S}_{\alpha^{\prime \prime} \gamma^{\prime \prime}}^{\alpha^{\prime} \gamma^{\prime}} & =\sum_{\alpha^{\prime} \beta^{\prime} \gamma^{\prime}} \underline{S}_{\alpha^{\prime} \gamma^{\prime}}^{\alpha \gamma}\left(q^{\prime}\right) \underline{S}_{\alpha^{\prime \prime} \beta^{\prime}}^{\alpha^{\prime} \beta}(q) \underline{R}_{\beta^{\prime \prime} \gamma^{\prime \prime}}^{-\beta^{\prime} \gamma^{\prime}}\left(q-q^{\prime}\right) .
\end{aligned}
$$

All other results remain unchanged. Yet, in order to obtain the right mixed YBE in Proposition 3.6, one needs to use the flip symmetry. In Lemma 3.16 , the proof of the second equivalence is valid only with this corrected definition. One should rewrite $S^{(m, n)}$ as follows:

$$
S^{(m, n)}=S_{1 \mid 1} S_{1 \mid 2} \cdots \cdots S_{1 \mid n} S_{2 \mid 1} \cdots S_{m \mid n},
$$

by exchanging the orders of factors which are acting on different tensor components. With this expression, one proves from the right mixed YBE that $\mathbb{1}_{\mathcal{H}_{+}^{\otimes m}} \otimes P_{R^{-}}^{(n)}$ commutes with $S^{(m, n)}$ for $m \geq 1, n \geq 2$.

The online version of the original article can be found under doi:10.1007/s00023-014-0337-1. 
Open Access. This article is distributed under the terms of the Creative Commons Attribution License which permits any use, distribution, and reproduction in any medium, provided the original author(s) and the source are credited.

Marcel Bischoff

Institut für Theoretische Physik

Universität Göttingen

Friedrich-Hund-Platz 1

37077 Göttingen, Germany

e-mail: bischoff@theorie.physik. uni-goettingen.de

Yoh Tanimoto

Graduate School of Mathematical Sciences

The University of Tokyo

Tokyo, Japan

e-mail: hoyt@ms.u-tokyo.ac.jp

and

Institut für Theoretische Physik

Universität Göttingen

Göttingen, Germany 